\title{
MAESTRI E TESTIMONI: PER UNA DIDATTICA DEL DIALOGO ECUMENICO NEL XXI SECOLO
}

\author{
MASTERS AND WITNESSES: FOR A TEACHING OF ECUMENICAL \\ DIALOGUE IN THE 21ST CENTURY
}

\section{MESTRES E TESTEMUNHAS: PARA UMA DIDÁTICA DO DIÁLOGO ECUMÊNICO NO SÉCULO XXI}

Marco Bontempi*

\section{RiASSUNTO}

Il dialogo ecumenico in Italia è diventato un tema centrale nella vita religiosa e sociale del XXI secolo a seguito della nuova situazione che si è creata con l'immigrazione di tanti cristiani non-cattolici, soprattutto dall'Europa Orientale. Il dialogo ecumenico si è sviluppato alla luce dell'esperienza di «maestri e testimoni» vissuti in Italia nel XX secolo, come Giorgio La Pira, a lungo sindaco di Firenze, del quale l'autore presenta la figura nella prospettiva della dimensione spirituale come elemento centrale per la promozione di una cultura del dialogo. Proprio figure come Giorgio La Pira hanno favorito la crescita di esperienza di dialogo nelle comunità locali che si sono dovute confrontare con pregiudizi nei confronti dell'altro, come l'autore mette in evidenza riportando un grafico con i dati sull'intolleranza in Italia.

Parole chiavi: Italia, Giorgio La Pira, Dialogo ecumenico, Dialogo islamo-cristiano, Insegnamento religioso, Intolleranza

\footnotetext{
* Professore ordinario all’Università di Firenze dove insegna sociologia generale; le sue numerose pubblicazioni affrontano teorie sociologiche del mutamento sociale, processi sociali di costruzione della condizione giovanile in Europa, mutamento valoriale e trasformazioni della cultura politica, sociologia della laicità e delle religioni e sociologia dell'educazione. Fa parte del Comitato di Redazione di Colloquia Mediterranea; è coordinatore del gruppo di Dialogo ebraico-cristiano-islamico di Firenze. E-mail: bontempi@unifi.it.
}

Artigo recebido em 01 de agosto de 2015 e aprovado em novembro de 2015 


\section{Abstract}

Ecumenical dialogue in Italy became a central topic in the religious and social life of the XXI century after the new situation created with the immigration of so many non-Catholic Christians, especially from Eastern Europe. The ecumenical dialogue is developed in the light of the "masters and witnesses" lived in Italy in the XX century, as Giorgio La Pira, mayor of Florence for the long time, whose the author presents the spiritual dimension as a central element for the promotion of a culture of dialogue. Figures as Giorgio La Pira favored the growth of experience of dialogue in the local communities which had to address the prejudice, as the author outlines quoting the tables on intolerance in Italy.

Keywords: Italia, Giorgio La Pira, Ecumenical Dialogue, Islamic-Christian Dialogue, Religious teaching, Intolerance

\section{RESUMO}

Na Itália, o diálogo ecumênico tornou-se um tema central na vida religiosa e social do XXI século, na sequência da nova situação criada com a imigração de tantos cristãos não católicos, sobretudo vidos do Leste Europeu. O diálogo ecumênico desenvolveu-se à luz da experiência de "mestres e testimunhas" que viveram na Itália, no século XX, como Giorgio La Pira, por muitos anos prefeito de Florença, de quem o autor apresenta a figura, na perspectiva da dimensão espiritual, como elemento central da promoção de uma cultura do diálogo. Personagens como Giorgio La Pira favoreceram o crescimento de experiências de diálogo nas comunidades locais, quando essas precisaram confrontar-se com preconceitos em relação ao outro, como o autor coloca em evidência, oferecendo um gráfico com os dados sobre o fenômeno da intolerância, na Itália.

Palavras-chave: Itália, Giorgio La Pira, Diálogo ecumênico, Diálogo islâmico-cristão, Ensino Religioso, Intolerância

\section{GIORGIO LA PIRA, ANTICIPATORE DEL DIALOGO DEL XXI SECOLO}

Giorgio La Pira ha avuto molte intuizioni profetiche. Il suo impegno politico e di testimonianza cristiana ha illuminato in modi diversi numerose questioni centrali per la comprensione dei "segni dei tempi" e le possibili risposte dei cristiani ${ }^{1}$. Tra queste il suo impegno e la sua riflessione sulle possibilità del dialogo ecumenico e anche tra le religioni abramitiche sono oggi particolarmente significativi e attuali.

In un'epoca dominata dalla guerra fredda come criterio di regolazione dei rapporti tra Stati nelle relazioni internazionali, La Pira ha sviluppato una visione del dialogo interreligioso come dimensione centrale di un paradigma delle relazioni internazionali

\footnotetext{
${ }^{1}$ Per informazioni approfondite sulla vita, l'opera e gli scritti di La Pira si veda www.giorgiolapira.org.
} 
connotato dalla ricerca non violenta della soluzione dei conflitti internazionali. In un'epoca dominata dall'idea che la laicità degli Stati comporti certamente separazione dalle chiese, ma molto spesso anche un atteggiamento di sospetto verso le religioni come elemento da cui le forme della politica moderna parevano aver preso congedo, La Pira ha portato dentro le relazioni internazionali il tema della religione non come ostacolo alla laicità degli Stati e fattore di conflitti, ma come risorsa centrale dello sviluppo di rapporti internazionali sempre più ricchi e complessi ad opera di una integrazione economica prima e politica poi, sempre più crescente.

Importanti, a proposito di dialogo interreligioso, furono i "viaggi-pellegrinaggi" di La Pira in Terra Santa, negli anni 1957-1960 e 1968. Le capitali di Giordania, Israele e Egitto furono visitate da La Pira in occasione del suo primo viaggio-pellegrinaggio in Terra Santa ed egli fu ricevuto dalle più alte autorità: Re Hussein di Giordania (dicembre 1957), il primo ministro israeliano Golda Meir (gennaio 1958), il presidente Nasser (gennaio 1960).

Nella sua appartenenza cattolica La Pira vedeva una responsabilità speciale della chiesa cattolica, una chiamata ad essere costruttrice di reti tra popoli e tra religioni, a mettere a servizio dell'umanità sofferente le proprie risorse di relazione e la propria diffusione planetaria.

L’importanza delle religioni nelle relazioni internazionali era affermata da La Pira come una conseguenza inseparabile da quel processo di avvicinamento dei popoli che oggi chiamiamo globalizzazione. Le religioni offrono la risorsa più importante per la critica dei cambiamenti sociali: la radice della spiritualità costituiva per lui una sorgente viva e un terreno fertile per la denuncia delle ideologie protagoniste della guerra fredda: il comunismo e il capitalismo. Nelle relazioni internazionali la conoscenza e l'incontro tra le fedi gli appariva come una risorsa che doveva essere ampiamente colta e impiegata da parte di chi davvero avesse voluto lavorare per la costruzione della pace.

I Colloqui mediterranei di Firenze furono un momento alto di questo percorso (CASTELLANI, 2009), mostrarono che il dialogo è possibile solo a partire dall'incontro e dalla conoscenza diretta. Quest'ultima è un potente antidoto agli stereotipi che le rappresentazioni ignoranti dell'atro religioso spesso producono. L'avvicinamento tra esponenti delle fedi abramitiche, e non solo, allora come oggi richiede di superare il formalismo dell'incontro e di una malintesa rappresentanza. Il dialogo ecumenico e interreligioso è indicato da La Pira come il luogo in cui cadono gli assunti impliciti sull'altro, gli stereotipi e i pregiudizi che secoli di separazione hanno prodotto. 
L’insegnamento di La Pira ci mostra che il dialogo ecumenico e interreligioso è una risorsa per lo sviluppo di visioni critiche della società e centrate sull'uomo come valore, proprio perché muovono dal primato di Dio. In questo senso la costruzione di relazioni internazionali di pace comporta che i popoli si avvicinino non solo perché mossi dalla ricerca di interessi politici ed economici, ma perché, superando gli stereotipi, possano davvero essere capaci di accoglienza e di condivisione. È in questa prospettiva che il dialogo ecumenico e interreligioso mostrano un inedito significato civico, perché rispondono alle profonde trasformazioni operate nella modernità e possono indicare la via di uno sviluppo che abbia a cuore la crescita di ogni donna e ogni uomo.

La Pira ha sostenuto questa visione del dialogo in un'epoca nella quale le differenze religiose nei paesi europei erano ancora soprattutto quelle delle divisioni storiche tra cristiani e tra cristiani ed ebrei. La sua visione del dialogo era profetica proprio perché era capace di indicarne il valore civico in un mondo che si apprestava a non essere più quello di un tempo. La sua idea di dialogo ecumenico e interreligioso è oggi straordinariamente attuale e lo rende a pieno titolo un testimone e un maestro del dialogo. Ciò che a mio parere è un elemento di valore è proprio l'importanza dell'incontro, l'importanza del superamento dei pregiudizi verso l'altro. Egli si è impegnato personalmente per lavorare in questa direzione. Oggi le diversità di fede, interne al cristianesimo e anche di altre religioni, sono presenti nella società come un dato di fatto. Il dialogo trova oggi molte condizioni e possibilità di sviluppo, ma ciò che appare ancora da superare sono proprio gli assunti impliciti sull'altro, i pregiudizi. È su questi aspetti minimi, pratici, ma fondamentali, che a mio parere l'insegnamento dell'ecumenismo può orientarsi per sviluppare una didattica davvero all'altezza dei tempi. 


\section{INSEGNARE L'ECUMENISMO COME PRATICA}

\subsection{Significati impliciti e didattica dell'ecumenismo}

Ciascuna tradizione ecclesiale ha sviluppato un proprio corpus dottrinale sull'importanza, la necessità, la natura, le condizioni del dialogo ecumenico. Protestanti e cattolici arrivano al dialogo muovendo da ragioni differenti e possono trovarsi a farlo avendo anche scopi differenti. Questo è un aspetto che ha molti riflessi sia nell'apprendimento da parte degli studenti, che anche nella pratica dei dialoghi fatti a livello delle comunità locali. Rispetto alla didattica dell'ecumenismo non solo è importante che questa non consista nella sola conoscenza delle ragioni della propria dottrina al dialogo ecumenico, ma è di estrema importanza che sia mirata allo sviluppo della capacità matetica di ristrutturare le condizioni dell'apprendimento nello studente attraverso la conoscenza delle differenti articolazioni dello stesso tema. Ad esempio sul tema dell'unità: la "nostalgia" cattolica di un'unità che coesiste e sussiste alle divisioni e che è motivazione all'ecumenismo (nei documenti conciliari: Lumen Gentium, Unitatis Redintegratio) a fronte dell'impegno protestante nel mostrare come in prospettiva storica e storico-sociale la diversità e la pluralità sia il fenomeno sempre presente e come la ricerca dell'unità non abbia senso come superamento delle differenze, ma sia una valorizzazione delle differenze (cfr il modello della comunione di comunioni).

Anche se dal punto di vista del lavoro teologico oggi vi è da parte cattolica e protestante una convergenza sul modello di unità Trinitaria inteso come koinonìa, resta - con la sua importanza - differente la logica di fondo: orientata alla tematizzazione attraverso categorie ontologiche da parte cattolica, orientata in prospettiva storico-sociale da parte protestante. Queste diversità nella tematizzazione sono rilevanti non solo per il lavoro teologico, ma, assunte in forma implicita e schematizzata, costituiscono anche quella che potremmo chiamare la mentalità ecclesiale propria di ciascuna delle diverse tradizioni del cristianesimo. Per mentalità ecclesiale intendo i significati che i membri di chiesa acquisiscono dalla loro partecipazione alla vita della chiesa, sia nelle forme liturgiche e del culto, ma anche e soprattutto nei modi di pensare e agire sviluppati nelle pratiche della socializzazione alla comunità locale di cui si sentono membri.

Si tratta di significati che sono elaborati collettivamente nelle pratiche ecclesiali quotidiane, che definiscono la forma di appartenenza a quella specifica comunità locale composta da quelle specifiche persone. Lo sfondo di significati della mentalità ecclesiale così 
intesa non è mai immediatamente ed esclusivamente derivabile dalla dottrina della chiesa e della tradizione cui quella comunità appartiene, ma, allo stesso tempo, non è mai indipendente dalla formulazione teologica della dottrina di quella tradizione. E' certamente connessa con il sapere teologico ecclesiale, che è portato nella comunità almeno dal prete o pastore. Però, i significati che connotano l'appartenenza alla comunità e la differenza con coloro che non ne fanno parte proprio perché condivisi (in assenza di conflitti interni) tendono a diventare ovvi, dati per scontati. E' su questo che la rappresentazione dell'altro, del diverso, può assumere i tratti dello stereotipo.

Molto spesso il lavoro teologico degli specialisti dell'ecumenismo ha effetti molto limitati sulla mentalità ecclesiale. Tale discrasia viene per lo più ricomposta sollecitando la speranza di effetti nei tempi lunghi del mutamento storico. È sicuramente vero che sui tempi lunghi le acquisizioni del lavoro teologico e le successive formulazioni dottrinali investono i fedeli e le loro maniere di interpretare l'appartenenza ecclesiale, ma le acquisizioni e le elaborazioni di questi elementi teologico-dottrinali non sono solo esito di questo processo topdown, ma interagiscono con dinamiche differenti e più ampie, come il mutamento culturale della società, che influenza e sollecita a sua volta il lavoro teologico. Questo scarto di piani e in particolare la mentalità ecclesiale, sopra accennata, dovrebbero essere tematizzati nella didattica dell'ecumenismo.

È importante considerare che la mentalità ecclesiale è presente anche nello studente come uno sfondo implicito che marca la sua appartenenza ecclesiale. Lo studio dell'ecumenismo dovrebbe poter favorire una presa di coscienza da parte dello studente dei significati impliciti con i quali pensa e definisce i cristiani che non appartengono alla propria chiesa. Far emergere gli assunti impliciti, dati per scontati, sui cristiani diversi da sé. Lo studio dell'ecumenismo offre un'importantissima occasione: può essere condotto in modo da promuovere nello studente uno sguardo sulla propria identità di fede che espliciti i significati assunti come impliciti, ovvi, dati per scontati. E similmente far affiorare e rendere espliciti i significati impliciti e dati per scontati relativi ai diversi (chi sono i cattolici per i protestanti e viceversa).

È chiaro che ciascuna tradizione ecclesiale cristiana ha elaborato ed elabora una propria comprensione e tematizzazione del dialogo ecumenico, delle sue ragioni e delle sue possibilità. Ciò fa si che nell'incontro con l'altro-cristiano possano esserci ragioni e idee di 
possibilità di direzioni di dialogo anche significativamente differenti tra i due interlocutori. In questo senso anche i manuali di ecumenismo si collocano lungo la linea della presentazione delle formulazioni dottrinali di una chiesa o tradizione cristiana, quella alla quale appartengono gli autori (e i loro lettori). Così ciascuna tradizione cristiana trasmette la propria dottrina ecumenica alle proprie nuove generazioni.

Ma il nucleo vitale dell'ecumenismo è muovere la riflessione e prendere posizione sulla questione della differenza e dell'alterità nella comprensione della fede e della vita ecclesiale cristiane. Ł̀ far questo come chiese, ma anche come singoli cristiani. Ł̀ la comprensione che peccato non sono solo le divisioni tra cristiani, ma anche, e non meno, le condanne reciproche. Questa riflessione e presa di posizione sono oggi necessarie come maturazione personale del singolo cristiano, come capacità di vivere in una società nella quale la questione dell'altro-religioso e della differenza tra valori e concezioni della fede è sempre più importante e anche sempre più intrecciata con conflitti e tensioni, oltre che con pratiche di dialogo e di cooperazione. Per questo la didattica dell'ecumenismo ha un compito specifico, più dell'insegnamento di altre discipline teologiche, quello di condurre lo studente ad una più matura consapevolezza dei propri assunti impliciti, dei propri stereotipi, delle proprie paure sull'altro-cristiano. Una via per far affiorare questi giudizi impliciti è di riflettere in senso pratico sul dialogo ecumenico come incontro e interazione tra due o più persone e da qui sviluppare alcuni elementi di stimolo per far affiorare i significati impliciti. In questo ci possono essere di aiuto le acquisizioni dell'interazionismo simbolico, una corrente della sociologia.

\subsection{Pensare il dialogo ecumenico come interazione}

Quali sono i prodotti del dialogo ecumenico? Abitualmente vengono indicati documenti, testi di convergenza o di accordo pieno. Insomma, testi prodotti in modo cooperativo. Accanto alla produzione di testi, ogni dialogo comporta l'interazione tra le persone che concretamente svolgono il dialogo. È noto quanto importante sia stata l'interazione tra attori ecumenici per favorire la stesura di testi ecumenici significativi. Meno ricordate sono le vicende nelle quali l'interazione ha ostacolato il raggiungimento di un accordo o la stesura di un testo comune. Spesso questi aspetti vengono considerati come "retroscena" o "informazioni di contesto" rispetto alla formulazione e stesura del contenuto del documento e come tali diventano oggetto del lavoro di ricostruzione condotto dagli storici dell'ecumenismo anche molti anni dopo. Eppure, per coloro che sono impegnati nel lavoro di dialogo, la dimensione 
dell'interazione costituisce un nucleo fondamentale dell'esperienza ecumenica, tanto per il raggiungimento di obiettivi condivisi, quanto per la crescita della fede personale. Solitamente si iscrive questa importantissima dimensione nella categoria dell'esperienza e in quanto tale la si ritiene estranea all'attività didattica. Ma se è impossibile insegnare l'esperienza e la maturazione della fede, sarebbe però molto importante orientare lo studente alla comprensione della dimensione pratica dell'interazione come un aspetto significativo del dialogo ecumenico.

Provo ad indicare qui alcuni elementi importanti, in parte tratti da un classico dell'analisi dell'interazione, che valgono per ogni situazione di interazione, ma che possono aiutare a pensare la situazione di dialogo come interazione.

Il primo elemento è relativo al lavoro di attribuzione dell'identità all'interlocutore, cioè la logica attraverso la quale vengono utilizzate le caratteristiche implicitamente pensate come tipiche di quella categoria di persone (ad es. "i cattolici sono...", "i protestanti sono...", “gli ortodossi sono...") per avviare l'interazione con l'interlocutore concreto che è di fronte. Qui non sono in gioco concetti teologici in senso stretto, ma gli usi sociali che vengono fatti dei concetti teologici nella definizione degli altri. Goffman ha descritto in modo illuminante questa logica:

«È la società a stabilire quali strumenti debbano essere usati per dividere le persone in categorie e quale complesso di attributi debbano essere considerati ordinari e naturali nel definire l'appartenenza ad una di quelle categorie. Sono i vari contesti sociali a determinare quali categorie di persone incontreremo, con maggiore probabilità, all'interno di tali contesti. La consuetudine sociale nei confronti di questi contesti stabiliti ci permette, senza una particolare attenzione o analisi approfondita, di instaurare un rapporto con le persone la cui presenza avevamo previsto. Quando ci troviamo davanti un estraneo, è probabile che il suo aspetto immediato ci consenta di stabilire in anticipo a quale categoria appartiene e quali sono i suoi attributi, qual è, in altri termini, la sua "identità sociale" (...). Ci fidiamo delle supposizioni che abbiamo fatto, le trasformiamo in aspettative normative e quindi in pretese inequivocabili.

È tipico non rendersi conto del fatto che siamo stati proprio noi a stabilire quei requisiti, quelle richieste, ed è altrettanto tipico che non siamo coscienti della loro natura finché non siamo costretti a decidere se corrispondono o no alla realtà. Solo allora è probabile che ci accorgiamo del fatto che, durante tutto il processo, ci siamo affidati a certi presupposti su come dovrebbe essere la persona che stiamo prendendo in considerazione. Per questo sarebbe più esatto definire i requisiti da noi fissati "richieste effettuali" e il carattere che attribuiamo alla persona un'attribuzione potenzialmente retrospettiva, una caratterizzazione "effettuale", una identità sociale virtuale. Chiameremo invece identità sociale attuale della persona la categoria a cui possiamo 
dimostrare che appartiene e gli attributi che è legittimo assegnargli» (GOFFMAN, 2003, p. 12).

Un secondo elemento, anche questo proprio di ogni tipo di interazione, è la territorialità personale, cioè il fatto che nell'interazione con altri, come scrive Goffman, «diveniamo vulnerabili all'intrusione delle loro parole e dei loro gesti nelle nostre riserve psichiche e diveniamo vulnerabili all'infrazione di quell'ordine espressivo che ci aspettiamo venga mantenuto in nostra presenza. Naturalmente, affermare che siamo resi vulnerabili in questi modi significa anche affermare che controlliamo le risorse necessarie per rendere gli altri vulnerabili allo stesso modo da parte nostra»(GOFFMAN, 2003, p. 49). Questa dinamica diviene particolarmente sensibile e rilevante nell'interazione di dialogo ecumenico (e interreligioso). L'ordine espressivo dell'interazione come cornice all'interno della quale gli interlocutori possano interagire - in altri termini, le sue regole base, "grammatica e sintassi" dell'interazione che vengono condivise non solo nei termini della loro applicazione, ma nella loro co-determinazione alla specifica situazione - è facilmente condizionato dalla categorizzazione che gli interlocutori fanno gli uni degli altri e dal tipo di conoscenza implicita e pregressa che gli interlocutori hanno delle categorie cui l'altro è riferibile. Questa dinamica di attribuzione dell'identità è di grande importanza nella costruzione di situazioni di dialogo ed è a mio parere fondamentale l'esserne almeno in parte consapevoli affinché l'ordine espressivo venga costruito in modo da favorire lo sviluppo del dialogo.

Nella didattica dell'ecumenismo con quali strumenti è possibile far affiorare i significati impliciti e le schematizzazioni dell'atro che ciascuno porta con sé? Un passaggio importante consiste nel dedicare del tempo a verbalizzare le proprie emozioni di fronte al diverso e al dialogo: paure dell'altro, paure di perdere se stessi, desiderio di assimilazione dell'altro per "farlo proprio", desiderio di trovare nell'altro la soluzione alle proprie frustrazioni, nel rapporto con la chiesa, nel rapporto con la dottrina, nel rapporto con Dio. Una seconda risorsa consiste nella realizzazione di incontri diretti tra gli studenti e cristiani di altre chiese, al fine di una conoscenza reciproca e anche di costruire relazioni personali a partire da elementi comuni (interessi, bisogni...) con contenuti talvolta solo parzialmente dottrinali, che consentano di sviluppare forme di interazione cooperativa.

In termini concreti tratterebbe di inserire nella didattica dell'ecumenismo alcune attività laboratoriali sia rivolte a sviluppare una maggiore consapevolezza dei propri assunti impliciti sull'identità ecclesiale propria e altrui, sia orientati alla conoscenza di persone concrete 
in situazioni concrete e incontri non occasionali. Il docente di ecumenismo aiuta in questo modo lo studente ad entrare in una rete di relazioni, a fare esperienza concreta del dialogo, a prendere almeno un po' consapevolezza dei propri pregiudizi sugli altri cristiani o delle proprie idealizzazioni. Infine, in termini esistenziali e spirituali sono elementi di grande importanza imparare a far sentire l'altro oggetto di un interesse sincero e l'esercizio dell'ascolto con la mente e il cuore, cioè la pedagogia di Dio, imparare da come Dio ci ascolta e accoglie.

Tutti questi elementi contribuiscono a delineare quel tipo di dialogo che in ambito ecumenico viene spesso definito come dialogo della vita, perché rivolto a coloro con i quali entriamo in contatto per le ragioni più differenti nell'ambito della vita quotidiana. Credo che nella formazione di ecumenisti sia importante certamente il fare esperienza del dialogo della vita, ma anche tematizzarlo in termini concettuali e farne motivo di riflessione e conoscenza consapevole, insomma di studio. Infatti, una formazione all'ecumenismo che si concentri esclusivamente sulla produzione teologica rischia di ignorare il vasto e complesso mondo dei significati impliciti e delle logiche dell'interazione e di dare una rappresentazione esclusivamente intellettuale di una realtà molto più ricca e complessa.

Tematizzare il dialogo della vita come ambito di studio e di formazione degli ecumenisti è sempre più necessario anche in ragione del cambiamento delle condizioni del dialogo ecumenico e interreligioso che è possibile osservare ormai da circa venti anni e che vedremo ulteriormente crescere nei prossimi anni. Si tratta dell'emergere di specifiche condizioni storico-sociali del dialogo ecumenico, e anche interreligioso. A queste vorrei ora dedicare alcune considerazioni.

\section{MINORANZE-MAGGIORANZA E IL RUOLO DIALOGO}

Da un punto di vista sociologico il dialogo ecumenico e anche quello interreligioso sono dialoghi asimmetrici tra minoranze e maggioranza. La percezione delle minoranze è un tema che occupa e occuperà spazio nella sfera pubblica, condizionando atteggiamenti e comportamenti collettivi, politici e non solo.

Tutte le chiese cristiane in Europa sono investite e influenzate in modi differenti, ma significativi, dai flussi migratori. Le identità religiose dei cristiani migranti portano forme di vivere la fede e pratiche religiose che impattano sulle forme delle chiese storiche europee. L'aumento delle presenze ortodosse al di fuori dei paesi tradizionali, il cambiamento delle 
chiese protestanti storiche europee per la presenza di immigrati protestanti che portano al loro interno modi differenti di vivere la fede, le reazioni ambivalenti della chiesa cattolica italiana: dal sostegno parziale alle chiese ortodosse, alla resistenza verso la presenza di presbiteri cattolico-orientali con la propria famiglia, ecc.

Queste trasformazioni influenzano la percezione pubblica della religione e dell'identità religiosa e rendono il dialogo ecumenico una modalità di rappresentazione pubblica di forme di intesa e di cooperazione che, indipendentemente dalla discussione sui temi teologici, ha grande influenza nella formulazione di un frame attraverso il quale interpretare le differenze religiose come opportunità di incontro e di accoglienza e non di conflitto. In questa prospettiva di costruzione di un frame dialogico e solidale, il dialogo ecumenico diventa un presupposto del dialogo interreligioso. Pur nelle dovute differenze, è importante che il dialogo interreligioso veda la testimonianza concorde delle diverse chiese cristiane. Solo così può essere plasmato un frame che possa in qualche modo contrastare le spinte conflittualiste nella interpretazione delle identità religiose e delle minoranze. I dati di una recentissima indagine del Pew Research Center sono preoccupanti, per l'Italia in modo particolare.

Grafico 1: Sentimento anti-minoranza verso Rom, Musulmani ed Ebrei in alcuni paesi europei $(\%)^{2}$

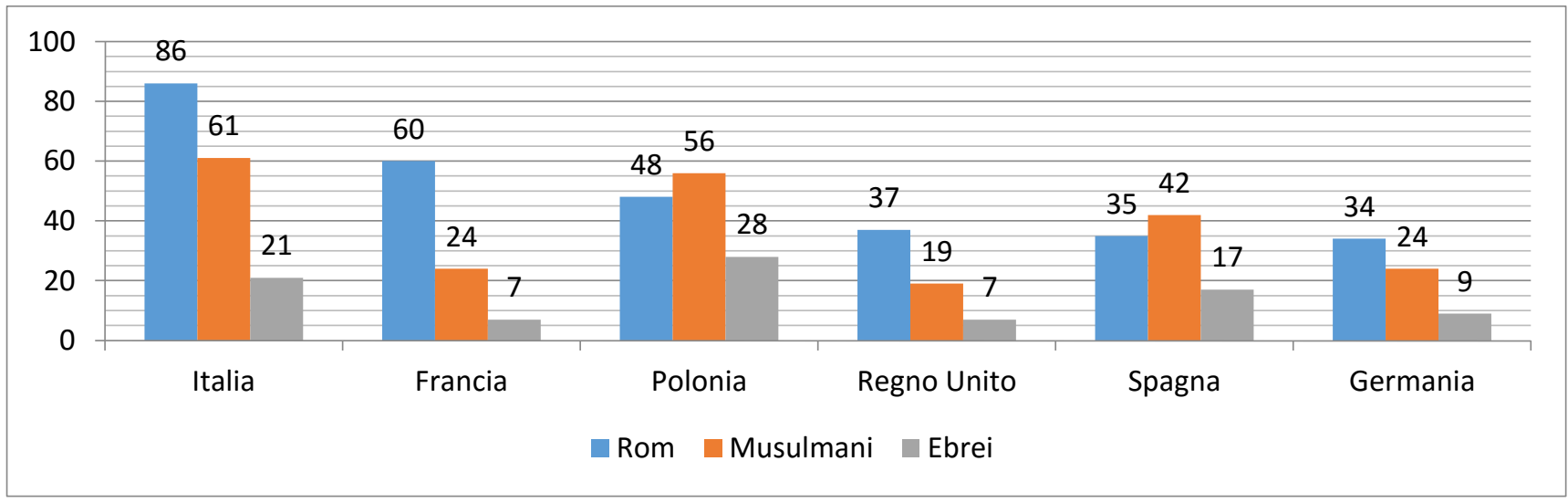

Grafico 2: Variazioni in 1 e 5 anni del sentimento anti-minoranza verso Rom, Musulmani ed Ebrei in Italia (\%) (STOKES, 2015, p. 34)

\footnotetext{
${ }^{2}$ Dati risultanti da 6.028 interviste a persone maggiori di 18 anni nel periodo 7/4 - 13/5 2015 (STOKES, 2015, p. $11)$.
} 


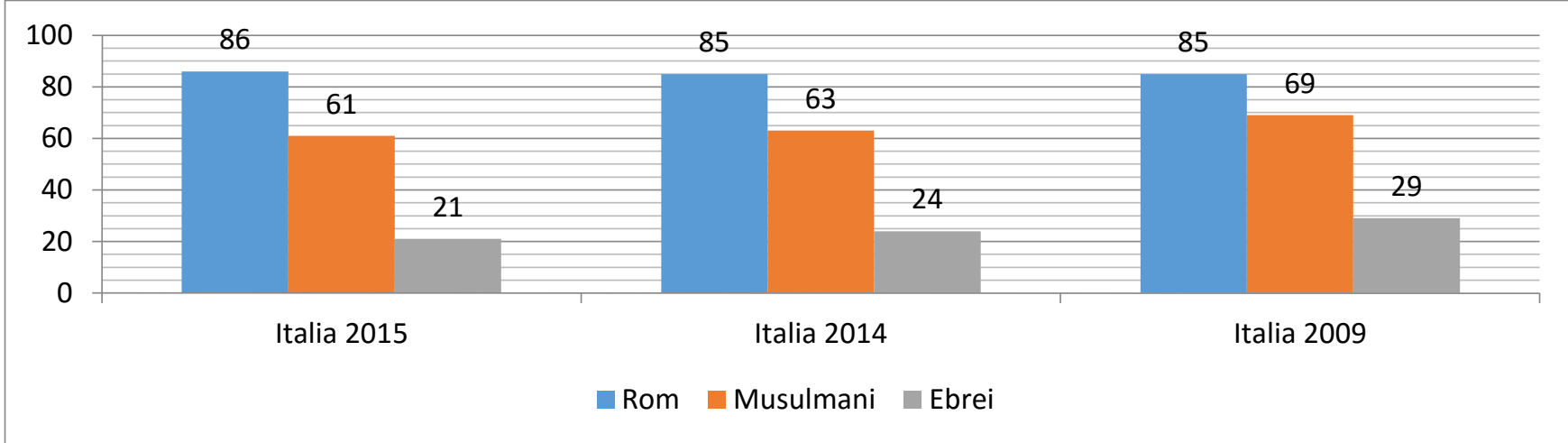

L'Italia emerge come il paese europeo che tra quelli studiati è connotato da un più forte sentimento di rifiuto e discriminazione verso tre minoranze che storicamente, per ragioni differenti, hanno subito e subiscono fortissime discriminazioni, anche su base religiosa. Questo sentimento di discriminazione è oggetto di poche variazioni nell'arco degli ultimi cinque anni, dimostrando quindi una stabilità e un radicamento che trova anche il suo versante politico in partiti xenofobi e razzisti che hanno ricevuto in passato e ricevono ancora oggi un consenso significativo da parte degli italiani.

In questo contesto il dialogo ecumenico e il dialogo interreligioso acquistano un valore civico e un'urgenza che i cristiani non possono trascurare, perché fare questo altro non sarebbe che negare il cuore del Vangelo e della testimonianza cristiana. Il dialogo ecumenico e interreligioso può davvero contribuire alla formazione di un senso civico per questa epoca di crisi. L'incontrare l'altro e il mostrare pubblicamente le possibilità di incontro e di cooperazione tra chiese cristiane e tra religioni abramitiche sono oggi la forma storicamente più significativa e necessaria di espressione pubblica della fede e del ruolo che le religioni possono svolgere per la civitas. Questa valenza civica del dialogo ecumenico ci riporta ai temi lapiriani accennati in apertura, con la differenza che negli anni '50-'60 La Pira formulava l'importanza dei dialogo tra le religioni del mediterraneo in una forma di consapevolezza profetica di un kairos che sarebbe stato prossimo. Oggi noi ci siamo dentro e siamo chiamati a saper reagire ai segni dei tempi. 


\section{RIFERIMENTI BIBLIOGRAFICI}

CASTELLANI, R. Giorgio La Pira e la pace. Il dialogo interreligioso nei "Colloqui Mediterranei". Pro Sanctitate, 2009

GOFFMAN, E. Stigma. Notes on the Management of Spoiled Identity. Simon \& Schuster: 1963 (tr.it. Stigma. L'identità negata. Ombre corte, 2003).

GOFFMAN, E. The Interaction order. American Sociological Review, Vol. 48, N. 1, Feb., 1983 (tr.it. L'ordine dell'interazione. Armando editore, 1998).

STOKES, B. Faith in European Project Reviving. Pew Research Center, 2015. 